\title{
MicroRNA-3666 suppresses the growth and migration of glioblastoma cells by targeting KDM2A
}

\author{
TAOTAO SHOU*, HUYIN YANG* ${ }^{*}$ JIA LV, DAI LIU and XIAOYANG SUN \\ Department of Neurosurgery, The Affiliated Huai'an No. 1 Hospital of Nanjing Medical University, \\ Huai'an, Jiangsu 223300, P.R. China
}

Received February 28, 2018; Accepted July 27, 2018

DOI: $10.3892 / \mathrm{mmr} .2018 .9698$

\begin{abstract}
MicroRNAs (miRNAs) are acknowledged as essential regulators in human cancer types, including glioblastoma (GBM). However, the functions of microRNA-3666 (miR-3666) in GBM remain unclear. In the present study, it was identified that the expression of miR-3666 was significantly downregulated in GBM tissues compared with adjacent normal tissues by reverse transcription-quantitative polymerase chain reaction. Additionally, miR-3666 was downregulated in GBM cell lines. Furthermore, it was observed that the miR-3666 expression level in patients with GBM was associated with prognosis. With functional experiments, it was identified that overexpression of miR-3666 significantly inhibited the proliferation, migration and invasion of GBM cells in vitro by Cell Counting kit- 8 and Transwell assays. Ectopic expression of miR-3666 significantly arrested GBM cells in the $G_{0}$ phase by fluorescence activated cell sorting. In terms of the underlying mechanism, it was identified that lysine-specific demethylase 2A (KDM2A) is a direct target of miR-3666 in GBM cells. Overexpression of miR-3666 significantly decreased the expression of KDM2A in GBM cells. Furthermore, it was observed that knockdown of KDM2A significantly suppressed the proliferation, migration and invasion of GBM cells. Collectively, the present results demonstrated that the miR-3666/KDM2A axis serves an important role in the progression of GBM, which provides novel insight into the development of therapeutic strategies for GBM treatment.
\end{abstract}

Correspondence to: Dr Xiaoyang Sun, Department of Neurosurgery, The Affiliated Huai'an No. 1 Hospital of Nanjing Medical University, 6 Beijing West Road, Huai'an, Jiangsu 223300, P.R. China

E-mail: sunxiaoyang0768@163.com

*Contributed equally

Key words: microRNA-3666, proliferation, migration, invasion, cell cycle progression, lysine-specific demethylase $2 \mathrm{~A}$, glioblastoma

\section{Introduction}

Glioblastoma (GBM) is the most malignant and frequent brain tumor, which is characterized as having high recurrence and poor outcome (1). At present, specific therapeutic strategies, including radiotherapy, surgery and chemotherapy, have been developed for GBM treatment (2). However, the outcomes of patients with GBM are very poor. At present, the 5-year overall survival rate of patients with GBM remains less than $3 \%$ (3). GBM has become a principal public health problem (4). Therefore, there it is imperative to search for novel therapeutic targets and develop efficient therapeutic approaches for GBM intervention.

MicroRNAs (miRNAs) are a class of short and non-coding RNAs, which have a length of $\sim 22$ nucleotides (5). Previous findings demonstrated that miRNAs are expressed in almost all types of cells and regulate gene expression by binding to the 3'-untranslated region (3'-UTR) of target mRNAs for degradation (6). In past decades, accumulating evidence suggests that miRNAs are involved in a diversity of biological processes, including cell proliferation, migration and survival (7-9). Due to their important physiological functions, dysregulation of miRNAs usually leads to human cancer, including GBM (10). Previous studies suggest that miRNAs may be promising biomarkers and therapeutic targets for tumor diagnosis, prognosis and treatment $(11,12)$. Improved understanding regarding the function and mechanism of miRNAs in GBM progression may contribute to the development of novel strategies for GBM treatment.

MicroRNA-3666 (miR-3666) has been demonstrated to inhibit the progression of non-small cell lung cancer (13) and thyroid carcinoma (14). Whether miR-3666 serves a role in GBM remains largely unknown. In the present study, it was identified that miR-3666 was significantly downregulated in GBM tissues and cell lines. In addition, the expression of miR-3666 expression levels is associated with the prognosis of patients with GBM. Furthermore, it was demonstrated that overexpression of miR-3666 significantly suppressed the proliferation, migration and invasion. Regarding the underlying mechanisms, it was observed that miR-3666 targeted lysine-specific demethylase 2A (KDM2A). By inhibition of KDM2A expression, miR-3666 decreased the proliferation, migration and invasion of GBM cells. Collectively, the present study, to the best of our knowledge, for the first time 
identified the key function of the miR-3666/KDM2A axis in GBM, which suggested that miR-3666 may be a promising therapeutic target for GBM intervention.

\section{Patients and methods}

Patient samples. The protocol of the present study and acquisition of tissue specimens was approved by the Biomedical Research Ethics Committee of The Affiliated Huaian No. 1 Hospital of Nanjing Medical University (Huai'an, China). A total of 38 GBM tissue specimens (21 males and 17 females; age range, 31-61 years old; median age, 46 years old) were obtained from patients who received surgical treatment at The Affiliated Huai'an No. 1 Hospital of Nanjing Medical University between September 2014 and October 2016. Patients who had previously undergone chemotherapy and radiotherapy were excluded. All enrolled patients signed a written informed consent document. To analyze overall survival, these samples were divided into two groups (miR-3666 low group and miR-3666 high group) according to the median value of miR-3666 expression.

Cell culture. The human GBM cell lines (A172, U251 and T98G) were obtained from the American Type Culture Collection (Manassas, VA, USA). Human astrocyte cell line HA was from Lonza Group, Ltd. (Basel, Switzerland). All cell lines were cultured in Dulbecco's modified Eagle's medium (DMEM; Sigma-Aldrich; Merck KGaA, Darmstadt, Germany) supplemented with $10 \%$ fetal bovine serum (FBS; Gibco; Thermo Fisher Scientific, Inc., Waltham, MA, USA), 100 U/ml penicillin $\mathrm{G}$ and $100 \mu \mathrm{g} / \mathrm{ml}$ streptomycin (Sigma-Aldrich; Merck KGaA). Normal human astrocytes (NHAs), obtained from Lonza Group, Ltd., were cultured in the provided astrocyte growth media and 5\% FBS. Cells were incubated in a humidified atmosphere with $5 \% \mathrm{CO}_{2}$ at $37^{\circ} \mathrm{C}$.

Transfection. The U251 cells $\left(2 \times 10^{6}\right)$ were seeded in 6-well plates and cultured for $18 \mathrm{~h}$ prior to transfection. Transfection was performed using Lipofectamine ${ }^{\circledR} 2000$ (Invitrogen; Thermo Fisher Scientific, Inc.), according to the manufacturer's protocol. A total of $48 \mathrm{~h}$ post-transfection, subsequent experiments were performed. RNA with no homology to any human genomic sequence was regarded as the negative control (miR-NC). The miR-3666 mimics (5'-CAGUGCAAGUGU AGAUGCCGA-3'; $50 \mathrm{nM}$ ) and small interfering (si)-KDM2A (5'-GCCAUCUUCCGUUGCAAAG-3'; $50 \mathrm{nM}$ ) sequences were designed and synthesized by Shanghai GenePharma Co., Ltd. (Shanghai, China).

Cell proliferation. For the Cell Counting kit-8 (CCK-8) assay, $2 \times 10^{3} \mathrm{U} 251$ cells per well were seeded in 96-well plates and the cells were subsequently cultured for 24,48 and $72 \mathrm{~h}$ prior to performing the CCK- 8 assay (Dojindo Molecular Technologies, Inc., Kumamoto, Japan). Following incubation with CCK- 8 at $37^{\circ} \mathrm{C}$, absorbance (optical density value) at a wavelength of $450 \mathrm{~nm}$ was detected and used to calculate cell viability.

Colony formation. A total of $2 \times 10^{3} \mathrm{U} 251$ cells per well were seeded into 6-well plates. Cells were cultured at $37^{\circ} \mathrm{C}$ for 14 days .
Formed clones were then fixed with $4 \%$ polyformaldehyde for $30 \mathrm{~min}$ at $25^{\circ} \mathrm{C}$, stained with $0.1 \%$ crystal violet for $20 \mathrm{~min}$ at $25^{\circ} \mathrm{C}$. Cell numbers in five random fields were counted using a light microscope (magnification, $\mathrm{x} 100$ ).

Cell cycle analysis. A total of $1 \times 10^{6}$ cells were harvested, washed twice with ice-cold PBS and then fixed in 70\% ethanol for $24 \mathrm{~h}$ at $4^{\circ} \mathrm{C}$. Cells were subsequently washed three times with ice cold PBS and incubated with $1 \mathrm{mg} / \mathrm{ml}$ RNase A (cat. no. R6148; Sigma Aldrich; Merck KGaA) for 30 min at $37^{\circ} \mathrm{C}$. Following this, cells were stained at $25^{\circ} \mathrm{C}$ for $10 \mathrm{~min}$ with $50 \mu \mathrm{g} / \mathrm{ml}$ propidium iodide (BD Biosciences, Franklin Lakes, NJ, USA) in $0.5 \%$ Tween-20 with PBS and subjected to analysis of cell cycle distribution using a BD FACScan flow cytometer (BD Biosciences) coupled with Cell Quest acquisition and analysis programs (version 2; BD Biosciences).

Bioinformatics analysis. The Target Scan tool (version 7.1; http://www.targetscan.org/index.html) was used to predict the potential targets of miR-3666.

Western blot analysis. U251 cells were lysed in cold radioimmunoprecipitation assay buffer (Thermo Fisher Scientific, Inc.), and the protein concentration was determined using a Bicinchoninic Acid Protein Assay kit (Pierce; Thermo Fisher Scientific, Inc.). Protein ( $40 \mu \mathrm{g} / \mathrm{lane})$ was separated via 10\% SDS-PAGE and then transferred to a polyvinylidene difluoride (PVDF) membrane (Thermo Fisher Scientific, Inc.). Following this, the membrane was blocked using 5\% non-fat milk in PBS (Thermo Fisher Scientific, Inc.) containing 0.1\% Tween-20 (Sigma-Aldrich; Merck KGaA) at room temperature for $3 \mathrm{~h}$. Subsequently, the PVDF membrane was incubated with rabbit anti-human polyclonal KDM2A (1:1,000; cat. no. ab191387; Abcam, Cambridge, MA, USA) and rabbit anti-human GAPDH (1:1,000; cat. no. ab9485; Abcam) primary antibodies at room temperature for $2 \mathrm{~h}$. Following washing with PBS for $10 \mathrm{~min}$, the PVDF membrane was incubated with horseradish peroxidase-tagged goat anti-rabbit secondary antibodies (1:5,000; cat. no. ab7090; Abcam) at room temperature for $1 \mathrm{~h}$. Membranes were then washed with PBS for $10 \mathrm{~min}$, and the protein bands were visualized using an Enhanced Chemiluminescence Western Blotting kit (Pierce; Thermo Fisher Scientific, Inc.), in accordance with the manufacturer's protocol. Protein densitometry was performed using ImageJ software (version 1.41; National Institutes of Health, Bethesda, MD, USA).

Transwell migration and Matrigel invasion assays. U251 cells were seeded in 24-well culture plates at a density of $1 \times 10^{5}$ cells/well and subsequently cultured at $37^{\circ} \mathrm{C}$ for $18 \mathrm{~h}$ prior to transfection. Lipofectamine ${ }^{\circledR} 2000$ RNAiMAX was added at a density of $1.5 \mu \mathrm{l} /$ well and either miR-3666 mimics or si-KDM2A was subsequently added ( $15 \mathrm{pM} /$ well). Cells were harvested $48 \mathrm{~h}$ post-transfection. Following the manufacturer's protocol, $2 \times 10^{4}$ cells with $100 \mu \mathrm{l}$ serum-free DMEM (Thermo Fisher Scientific, Inc.) were seeded into the upper chamber of the Transwell plates (Costar; Corning Incorporated, Corning, NY, USA) for the migration assays, whereas, cells with $100 \mu \mathrm{l}$ serum-free DMEM were plated into the upper chamber of an insert coated with Matrigel (BD Biosciences, Franklin Lakes, NJ, USA) for the invasion assays. The lower chambers were 
A

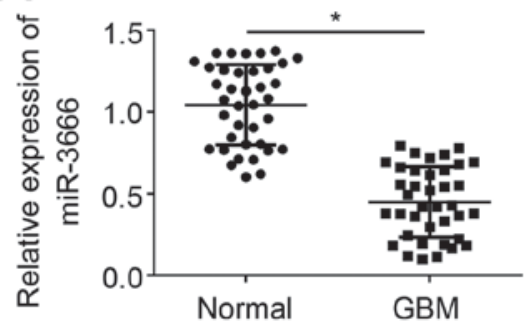

$\mathrm{B}$

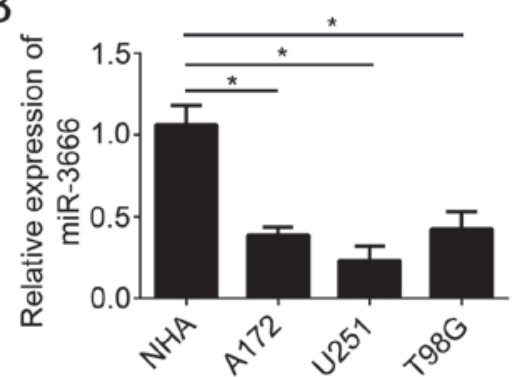

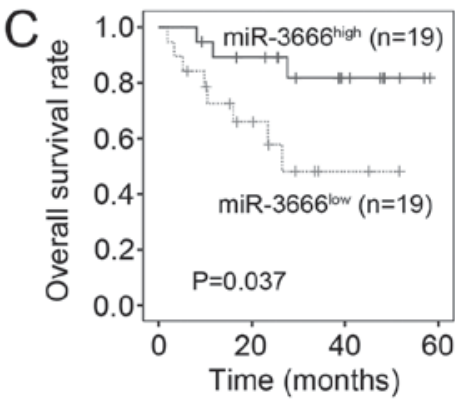

Figure 1. miR-3666 is downregulated in GBM tissues. (A) Reverse transcription-quantitative polymerase chain reaction analysis indicated that miR-3666 was downregulated in GBM tissues compared with adjacent normal tissues. (B) Relative expression of miR-3666 in GBM cell lines and NHA cells. (C) Kaplan-Meier survival analysis based on miR-3666 expression levels in GBM tissues. ${ }^{*}<0.05$. All data are representative of three independent experiments and are expressed as the mean \pm standard deviation. GBM, glioblastoma; miR, microRNA; NHA, normal human astrocytes.

filled with $600 \mu$ l DMEM containing 10\% FBS. Following $48 \mathrm{~h}$ of incubation, the cells remaining on the upper membrane were removed with cotton swabs, whereas, those that had migrated or invaded through the membrane were fixed in $4 \%$ polyformaldehyde for $30 \mathrm{~min}$ at $25^{\circ} \mathrm{C}$ and stained with $0.1 \%$ crystal violet for $20 \mathrm{~min}$ at $25^{\circ} \mathrm{C}$. The number of cells was calculated by imaging five random fields/filters using a fluorescence inversion microscope system (Nikon Corporation, Tokyo, Japan) at a magnification of x200. All the experiments were performed at least three times independently.

Reverse transcription-quantitative polymerase chain reaction $(R T-q P C R)$. Total RNA of GBM samples and cultured cells were extracted with TRIzol ${ }^{\circledR}$ reagent (Invitrogen; Thermo Fisher Scientific, Inc.), according to the manufacturer's protocol. cDNA was synthesized from isolated RNA using a TaqMan MicroRNA Reverse Transcription kit (Applied Biosystems; Thermo Fisher Scientific, Inc.) according to the manufacturer's protocol. qPCR was performed with a TaqMan MicroRNA Assay kit (Applied Biosystems; Thermo Fisher Scientific, Inc.) on an ABI7300 PCR detection system. The thermocycling conditions were as follows: Denaturation at $95^{\circ} \mathrm{C}$ for $10 \mathrm{~min}$; followed by 40 cycles of denaturation at $95^{\circ} \mathrm{C}$ for $15 \mathrm{sec}$ and elongation at $60^{\circ} \mathrm{C}$ for $1 \mathrm{~min}$. The expression levels of miR-3666 and KDM2A were normalized with U6 or GAPDH. The RT-qPCR primer sequences were as follows: miR-3666 forward, A5'-ACG AGACGACGACAGAC-3' and reverse, 5'-CAGTGCAAGTGT AGATGCCGA-3'; U6 forward, 5'-AACGAGACGACGACA GAC-3' and reverse, 5'-GCAAATTCGTGAAGCGTTCCA TA-3'; KDM2A forward, 5'-GTGACGCAGCAGCATTGT TC-3'; and reverse, 5'-CAGACTACCCAGAGGGAGCA-3'; and GAPDH forward, 5'-ATGTTGCAACCGGGAAGGAA-3' and reverse, 5'-AGGAAAAGCATCACCCGGAG-3'. Quantification was performed using the $2^{-\triangle \triangle \mathrm{Cq}}$ method (15).

Luciferase reporter assay. To investigate whether downregulation of KDM2A by miR-3666 is caused by the direct interaction between the miRNA seed sequence and the 3'UTR of the target mRNA, pmirGLO dual-luciferase vectors (cat. no. E133A; Promega, Corporation, Madison, WI, USA) containing the 3'UTR of KDM2A mRNA were constructed, pmirGLO-KDM2A 3'UTR. Two individual vectors were prepared, containing wild-type (WT) or mutated seed region sequences of the miR-3666-binding site in the 3'UTR of
KDM2A mRNA. U251 cells were transfected with NC or miR-3666 mimics in addition to either WT or mutant-type luciferase vector using Lipofectamine ${ }^{\circledR} 2000$ (Invitrogen; Thermo Fisher Scientific, Inc.). A total of $48 \mathrm{~h}$ post-transfection, the effect of the miR-3666 on luciferase expression was assessed using a Dual-GLO ${ }^{\mathrm{TM}}$ Luciferase Assay System (cat. no. E2940; Promega, Corporation). Luciferase activity was normalized to Renilla luciferase activity.

Statistical analysis. Each experiment was repeated at least three times. Data are expressed as the mean \pm standard deviation. All statistical analyses were performed using SPSS 20.0 (IBM Corp., Armonk, NY, USA) and GraphPad Prism (version 6; GraphPad Software, Inc., La Jolla, CA, USA). The Kaplan-Meier method was used to calculate the survival curve, and log-rank test to determine statistical significance. Student's t-test and one-way analysis of variance followed by Tukey's post hoc test were used to analyze two or multiple groups, respectively, for statistical significance. $\mathrm{P}<0.05$ was considered to indicate a statistically significant difference.

\section{Results}

miR-3666 is downregulated in GBM tissues. miRNAs have been demonstrated to be important regulators in human cancer. In order to examine the function of miR-3666 in GBM, its expression patterns were analyzed by RT-qPCR. The results demonstrated that miR-3666 expression was significantly decreased in GBM tissues $(n=38)$ compared with adjacent normal tissues ( $\mathrm{n}=38$; Fig. $1 \mathrm{~A}$; $\mathrm{P}<0.05)$. The expression of miR-3666 was additionally assessed in GBM cell lines. RT-qPCR analysis demonstrated that miR-3666 expression was significantly downregulated in U251, A172 and T98G cells compared with NHAs (Fig. 1B; P<0.05). Furthermore, to determine whether miR-3666 expression is able to serve as a biomarker for GBM prognosis, these GBM samples were divided into two groups based on miR-3666 expression levels. Kaplan-Meier survival analysis revealed that higher expression of miR-3666 in patients with GBM was associated with higher survival rate (Fig. 1C). Collectively, these results demonstrated miR-3666 was downregulated in GBM tissues, which suggested its dysregulation may contribute to GBM progression. 

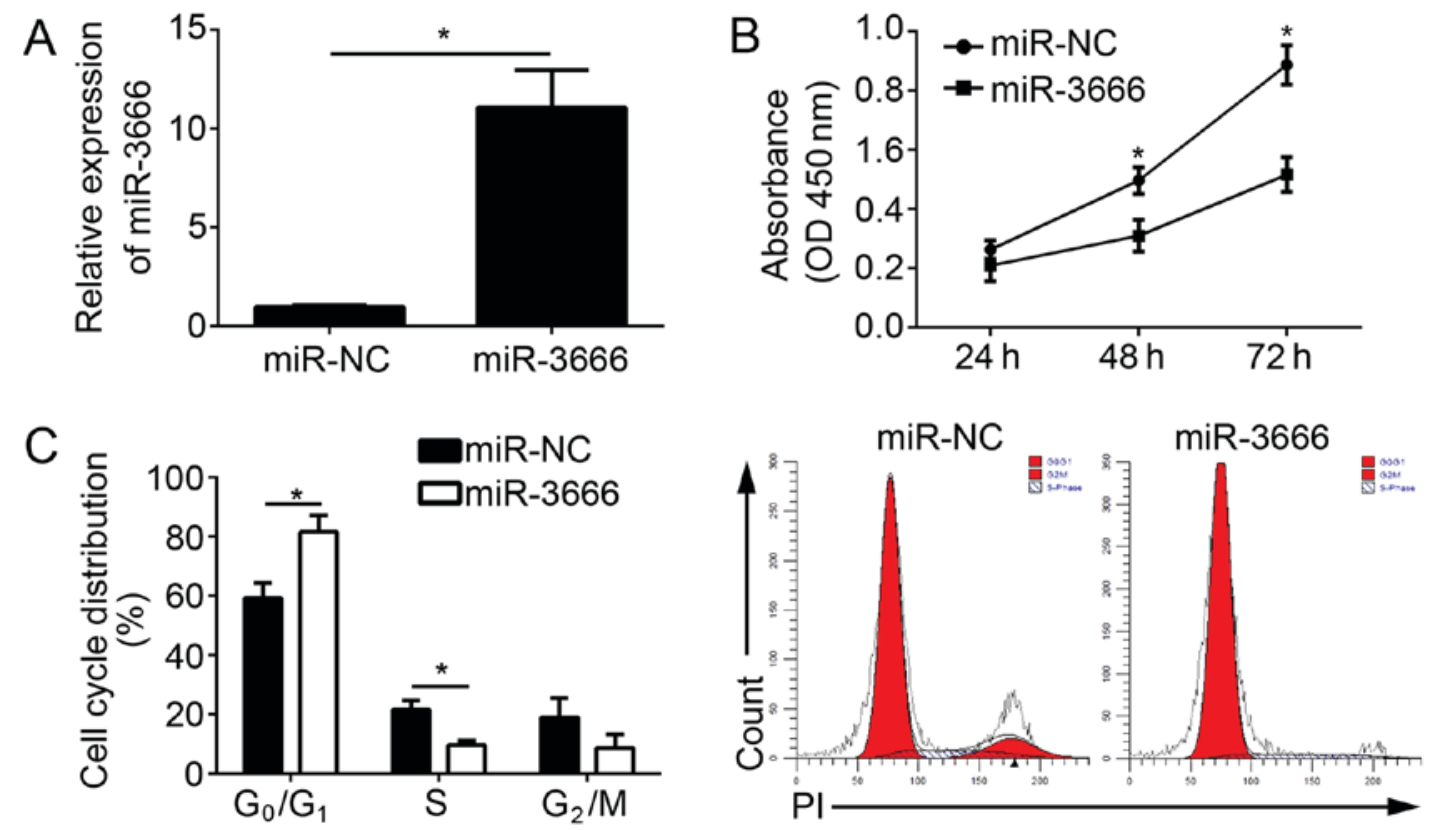

Figure 2. miR-3666 suppresses GBM cell proliferation and arrests cell cycle progression. (A) Relative expression of miR-3666 in U251 cells transfected with miR-NC or miR-3666 mimics. (B) Cell Counting kit-8 assays were performed with U251 cells transfected with miR-NC or miR-3666 mimics. (C) Cell cycle distribution was measured in U251 cells by fluorescence-activated cell sorting analysis. " $\mathrm{P}<0.05$. All data are representative of three independent experiments and are expressed as the mean \pm standard deviation. GBM, glioblastoma; miR, microRNA; NC, negative control; OD, optical density; PI, propidium iodide.

A

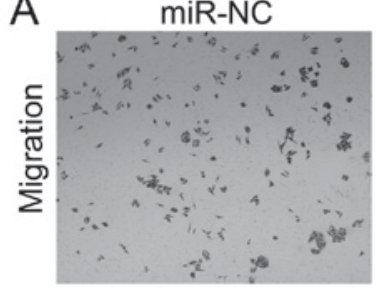

C

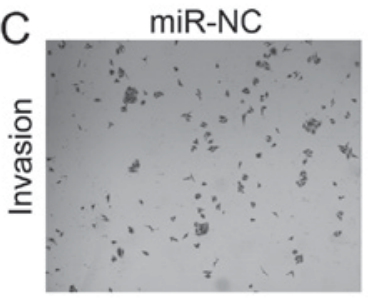

$\mathrm{miR}-3666$

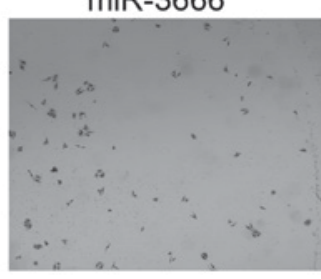

$\operatorname{miR}-3666$

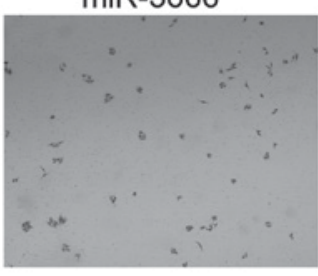

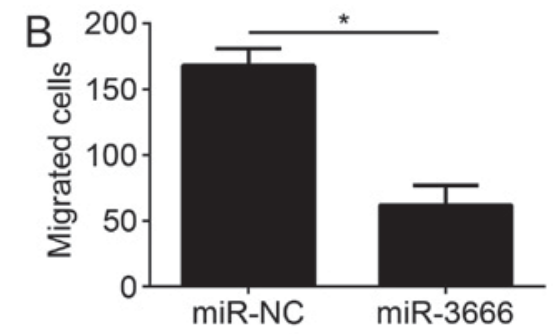

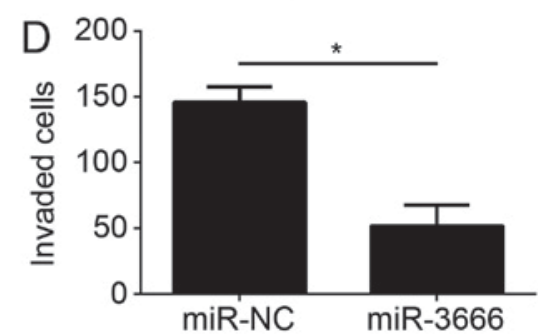

Figure 3. miR-3666 inhibits the migration and invasion of glioblastoma cells. (A-D) Transwell migration and invasion assays were used to determine the effect of miR-3666 on cellular migration and invasion (magnification, $\mathrm{x} 100$ ). ${ }^{*} \mathrm{P}<0.05$. All data are representative of three independent experiments and are expressed as the mean \pm standard deviation. miR, microRNA; NC, negative control.

miR-3666 suppresses GBM cell proliferation and cell cycle progression. To determine the physiological roles of miR-3666 in GBM, miR-3666 was overexpressed in U251 cells by transfection with miR-3666 mimics. The RT-qPCR results indicated that miR-3666 was significantly upregulated in U251 cells transfected with miR-3666 mimics compared with the miR-NC group (Fig. $2 \mathrm{~A} ; \mathrm{P}<0.05$ ). CCK-8 assays were conducted to analyze the effect of miR-3666 on cell proliferation. The results demonstrated that the overexpression of miR-3666 suppressed the proliferation of U251 cells (Fig. 2B) and decreased the number of colonies (data not shown). To determine whether impaired proliferation by miR-3666 was induced by an aberrant cell cycle, the cell cycle distribution in
U251 cells was measured by fluorescence-activated cell sorting (FACS). The results demonstrated that the overexpression of miR-3666 significantly increased the cells in $G_{0} / G_{1}$ phase and significantly decreased the number of cells in the $\mathrm{S}$ phase (Fig. 2C; $\mathrm{P}<0.05$ ). In conclusion, the data suggested that miR-3666 suppressed the proliferation and cell cycle progression of GBM cells.

miR-3666 inhibits the migration and invasion of GBM cells. Tumor metastasis is a principal cause of malignancy. Therefore, the effect of miR-3666 on GBM cell migration and invasion was analyzed. Transwell migration and Matrigel invasion assays were conducted with U251 cells transfected with 
A
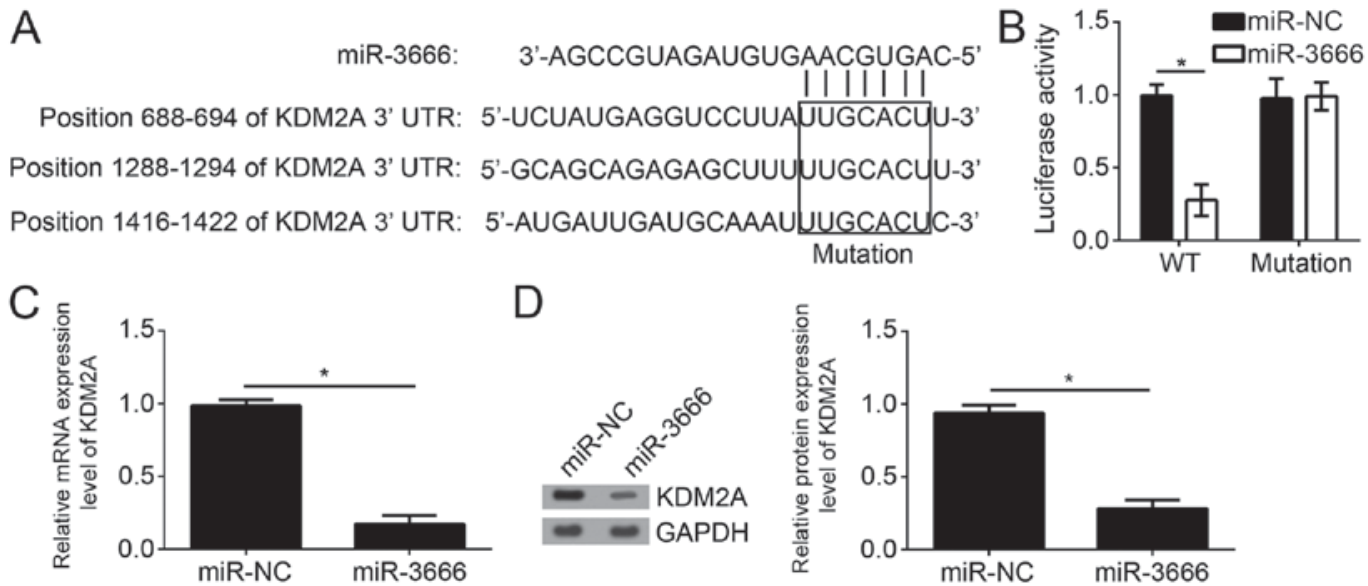

Figure 4. KDM2A is a target of miR-3666. (A) Diagram for miR-3666 binding sites in the 3'-UTR of KDM2A mRNA. (B) miR-3666 overexpression suppressed the luciferase activity in U251 cells transfected with WT 3'-UTR of KDM2A mRNA. (C) Reverse transcription-quantitative polymerase chain reaction and (D) western blot analysis were used to analyze the effect of miR-3666 on KDM2A mRNA and protein expression levels in U251 cells. * $<0.05$. A11 data are representative of three independent experiments and are expressed as the mean \pm standard deviation. KDM2A, lysine-specific demethylase $2 \mathrm{~A}$; miR, microRNA; 3'-UTR, 3'-untranslated region; WT, wild-type.

miR-NC or miR-3666. The results demonstrated that ectopic expression of miR-3666 significantly decreased the number of migrated and invaded cells (Fig. 3A; $\mathrm{P}<0.05$ ).

KDM2A is a target of miR-3666. To further examine the mechanism of miR-3666, the target genes of miR-3666 were searched for in GBM cells. By bioinformatics analysis using TargetScan7 (http://www.targetscan.org/vert_71/), it was identified that KDM2A was one of the most potential targets. There are three potential binding sites of miR-3666 in the 3'-UTR region of KDM2A (Fig. 4A). To verify the direct interaction between miR-3666 and KDM2A mRNA, luciferase reporter assays were performed with the reporter plasmid containing the WT or mutant 3'-UTR region of KDM2A mRNA. The results suggested that overexpression of miR-3666 significantly decreased the luciferase activity in U251 cells transfected with WT reporter plasmid; however, not the mutant plasmid (Fig. 4B; P<0.05). Furthermore, it was observed that overexpression of miR-3666 significantly downregulated the mRNA expression level of KDM2A in U251 cells (Fig. 4C; $\mathrm{P}<0.05$ ). Consistently, western blot analysis additionally suggested that miR-3666 overexpression significantly inhibited the protein expression level of KDM2A in U251 cells (Fig. 4D; $\mathrm{P}<0.05$ ).

Knockdown of KDM2A suppresses the proliferation, migration and invasion of GBM cells. To determine whether KDM2A is important in miR-3666-mediated inhibition of GBM cell proliferation, migration and invasion, the effect of KDM2A on these activities in U251 cell was investigated. Upon transfection with specific siRNA against KDM2A, the KDM2A expression level was effectively knocked down in U251 cells (Fig. 5A). CCK-8 assays demonstrated that knockdown of KDM2A suppressed the proliferative ability of U251 cells (Fig. 5B). FACS analysis suggested that KDM2A knockdown additionally resulted in a significant decrease of the cells in $\mathrm{S}$ phase and a significant increase of cells in the $\mathrm{G}_{0} / \mathrm{G}_{1}$ phase (Fig. $5 \mathrm{C}$; $\mathrm{P}<0.05)$. Furthermore, Transwell and Matrigel assays indicated that KDM2A depletion significantly decreased the abilities of migration and invasion in U251 cells (Fig. 5D and E; P<0.05).
Collectively, these results suggested that KDM2A knockdown inhibited the proliferation, migration and invasion of GBM cells, which suggested that miR-3666 regulates GBM progression by targeting KDM2A at least partially.

\section{Discussion}

The present results demonstrated that miR-3666 serves as a tumor suppressor in GBM cells. Additionally, it was identified that KDM2A may be a direct target of miR-3666 in U251 cells. Overexpression of miR-3666 or knockdown of KDM2A suppressed the proliferation, migration and invasion, which suggested that miR-3666 suppressed the progression of GBM by targeting KDM2A at least in part.

miRNAs are a group of short regulatory non-coding RNAs that participate in the regulation of post-transcriptional gene expression by binding to complementary sequences (16). Accumulating evidence suggested that miRNAs are involved in various cell processes, including cell proliferation, apoptosis, migration and invasion (17). Dysregulation of miRNAs is reported in human cancer, including colorectal cancer (18), glioma (19), cholangiocarcinoma (20), breast cancer (21), non-small cell lung cancer (22), esophageal squamous carcinoma (23), gastric cancer (24), papillary thyroid cancer (25) and renal cell carcinoma (26). An increasing number of studies suggest that miRNAs are potential biomarkers for cancer diagnosis and prognosis, and effective therapeutic targets for tumor treatments (26-28). Numerous miRNAs have been reported to regulate the development and progression of GBM (27). For example, Wang et al (10) reported that miRNA-598 inhibits cell proliferation and invasion of GBM by directly targeting metastasis associated in colon cancer-1. In addition, Chen et al (28) demonstrated that downregulation of miR-205 is associated with GBM cell migration, invasion and the epithelial-mesenchymal transition, by targeting zinc finger E-box-binding homeobox 1 via the protein kinase B/mammalian target of rapamycin signaling pathway. miR-3666 has been demonstrated to inhibit the progression of non-small cell lung cancer (13) and thyroid carcinoma (14). However, its 
A
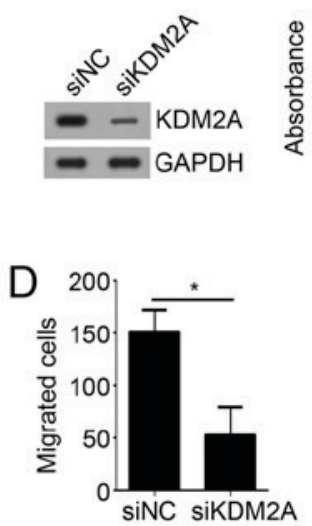

B

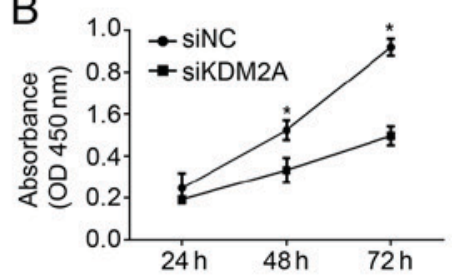

siNC

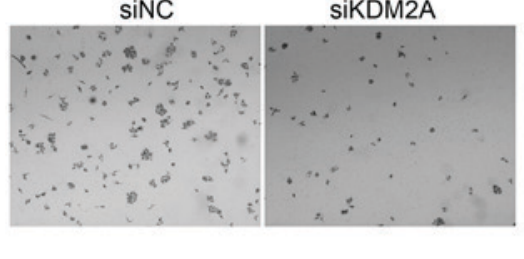

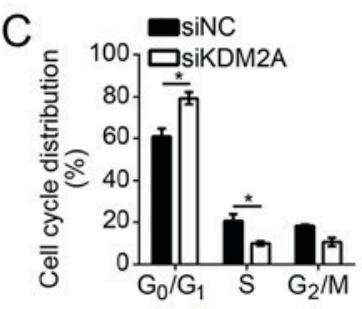

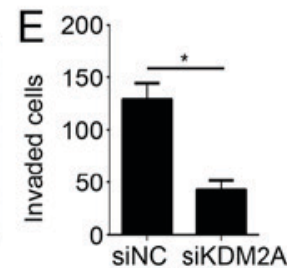

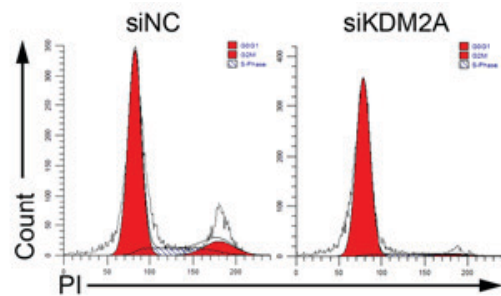

siNC

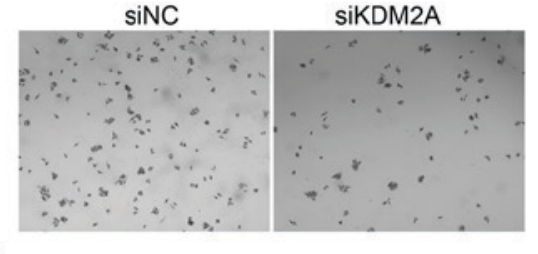

Figure 5. Knockdown of KDM2A suppresses the proliferation, migration and invasion of GBM cells. (A) Western blotting was conducted to analyze the protein levels of KDM2A in U251 cells transfected with siNC or siKDM2A. (B) Cell Counting kit-8 assays were used to determine cell proliferation. (C) Cell cycle distribution was measured by fluorescence-activate cell sorting. (D) Migration and (E) invasion potentials of U251 cells were measured by Transwell and Matrigel assays, respectively (magnification, x100). ${ }^{*} \mathrm{P}<0.05$. All data are representative of three independent experiments and are expressed as the mean \pm standard deviation. KDM2A, lysine-specific demethylase 2A; GBM, glioblastoma; si, small interfering; NC, negative control; OD, optical density; PI, propidium iodide.

exact role and underlying mechanisms of miR-3666 in GBM require investigation. In the present study, it was observed that miR-3666 was significantly downregulated in GBM tissues. Furthermore, it was demonstrated that overexpression of miR-3666 inhibited cell proliferation, migration and invasion, and arrested cell cycle progression in U251 cells.

KDM2A is a histone $\mathrm{H} 3$ lysine 36 demethylase and consists of an F-box, a JmjC domain, a CXXC zinc finger, a PHD domain and three leucine-rich repeat elements (29). A previous study suggested that KDM2A exerts an oncogenic role in a number of cancer types (29). Chen et al (30) demonstrated that KDM2A represses TET2 to increase DNA methylation and dowregulation of tumor suppressor genes in breast cancer. Huang et al (31) reported that KDM2A enhanced tumor cell growth and migration in gastric cancer. In addition, Wagner et al (32) suggested that KDM2A promotes lung tumorigenesis by epigenetically enhancing extracellular signal-regulated kinase $1 / 2$ signaling. However, the function of KDM2A in GBM has not been defined. In the present study, it was identified that KDM2A is a direct target of miR-3666 in GBM cells. It was demonstrated that overexpression of miR-3666 significantly decreased the mRNA and protein expression levels of KDM2A in U251 cells. Furthermore, with functional experiments, it was observed that KDM2A silencing significantly suppressed the proliferation, migration and invasion of U251 cells, which suggested that KDM2A is responsible for the function of miR-3336 in GBM progression at least in part.

In conclusion, the present study, to the best of our knowledge, for the first time identified the function of miR-3666 in GBM progression, and demonstrated that miR-3666 inhibited the proliferation, migration and invasion of GBM cell by targeting KDM2A. The present study suggests that miR-3666 may be a promising therapeutic target for GBM treatment. However, there were several limitations in the present study. Whether miR-3666 has an effect on GBM cell apoptosis was not investigated. Furthermore, the present study did not use in vivo assays, which are better able to demonstrate the roles of miR-3666 in GBM. Future studies should investigate how KDM2A participates in the regulation of GBM progression.

\section{Acknowledgements}

Not applicable.

\section{Funding}

No funding was received.

\section{Availability of data and materials}

The datasets used and/or analyzed during the current study are available from the corresponding author on reasonable request.

\section{Authors' contributions}

TS, HY and XS contributed to the conception and design of the present study, analyzed and interpreted the data, and wrote the manuscript. JL and DL conducted the experiments. All authors read and approved the final manuscript.

\section{Ethics approval and consent to participate}

For the use of human samples, the protocol for the present study was approved by the Institutional Ethics Committee of The Affiliated Huai'an No. 1 Hospital of Nanjing Medical University and all enrolled patients signed a written informed consent document.

\section{Patient consent for publication}

All patients within the present study provide consent for the publication of their data. 


\section{Competing interests}

The authors declare that they have no competing interests.

\section{References}

1. Zhao F, Li M, Kong L, Zhang G and Yu J: Delineation of radiation therapy target volumes for patients with postoperative glioblastoma: A review. Onco Targets Ther 9: 3197-3204, 2016

2. Koekkoek JA, Kerkhof M, Dirven L, Heimans JJ, Reijneveld JC and Taphoorn MJ: Seizure outcome after radiotherapy and chemotherapy in low-grade glioma patients: A systematic review. Neuro Oncol 17: 924-934, 2015.

3. Bradshaw A, Wickremsekera A, Tan ST, Peng L, Davis PF and Itinteang T: Cancer stem cell hierarchy in glioblastoma multiforme. Front Surg 3: 21, 2016.

4. Neagu MR and Reardon DA: An update on the role of immunotherapy and vaccine strategies for primary brain tumors. Curr Treat Options Oncol 16: 54, 2015.

5. Li X, Nie J, Mei Q and Han WD: MicroRNAs: Novel immunotherapeutic targets in colorectal carcinoma. World J Gastroenterol 22: 5317-5331, 2016.

6. Diab M, Muqbil I, Mohammad RM, Azmi AS and Philip PA: The role of microRNAs in the diagnosis and treatment of pancreatic adenocarcinoma. J Clin Med 5: pii: E59, 2016

7. Song H, Rao Y, Zhang G and Kong X: MicroRNA-384 inhibits the growth and invasion of renal cell carcinoma cells by targeting astrocyte elevated gene 1. Oncol Res: Aug 25, 2017 (Epub ahead of print).

8. Anninos P, Chatzimichael A, Adamopoulos A, Kotini A and Tsagas N: A combined study of MEG and pico-Tesla TMS on children with autism disorder. J Integr Neurosci 15: 497-513, 2016.

9. Wang X, Qi G, Zhang J, Wu J, Zhou N, Li L and Ma J: Knockdown of long noncoding RNA small nucleolar RNA hos gene 12 inhibits cell growth and induces apoptosis by upregulating miR-138 in nonsmall cell lung cancer. DNA Cell Biol 36: 892-900, 2017

10. Wang N, Zhang $\mathrm{Y}$ and Liang $\mathrm{H}$ : microRNA-598 inhibits cell proliferation and invasion of glioblastoma by directly targeting metastasis associated in colon cancer-1. Oncol Res: Feb 14, 2018 (Epub ahead of print).

11. Hamam R, Hamam D, Alsaleh KA, Kassem M, Zaher W, Alfayez M, Aldahmash A and Alajez NM: Circulating microRNAs in breast cancer: Novel diagnostic and prognostic biomarkers. Cell Death Dis 8: e3045, 2017.

12. Ren J, Yang Y, Xue J, Xi Z, Hu L, Pan SJ and Sun Q: Long noncoding RNA SNHG7 promotes the progression and growth of glioblastoma via inhibition of miR-5095. Biochem Biophys Res Commun 496: 712-718, 2018.

13. Shi H, Ji Y,Zhang D, Liu Y and Fang P: MicroRNA-3666-induced suppression of SIRT7 inhibits the growth of non-small cell lung cancer cells. Oncol Rep 36: 3051-3057, 2016.

14. Wang G, Cai C and Chen L: MicroRNA-3666 regulates thyroid carcinoma cell proliferation via MET. Cell Physiol Biochem 38: 1030-1039, 2016

15. Livak KJ and Schmittgen TD: Analysis of relative gene expression data using real-time quantitative PCR and the 2(-Delta Delta C(T)) method. Methods 25: 402-408, 2001.

16. Duan J, Zhou K, Tang X, Duan J and Zhao L: MicroRNA-34a inhibits cell proliferation and induces cell apoptosis of glioma cells via targeting of Bcl-2. Mol Med Rep 14: 432-438, 2016.

17. Hua S, Liu C, Liu L and Wu D: miR-142-3p inhibits aerobic glycolysis and cell proliferation in hepatocellular carcinoma via targeting LDHA. Biochem Biophys Res Commun 496: 947-954, 2018.
18. Liu Y, Chen X, Cheng R, Yang F, Yu M, Wang C, Cui S, Hong Y, Liang $\mathrm{H}$, Liu M, et al: The Jun/miR-22/HuR regulatory axis contributes to tumourigenesis in colorectal cancer. Mol Cancer 17: 11, 2018.

19. Sun G, SiMa G, Wu C, Fan Y, Tan Y, Wang Z, Cheng G and Li J: Decreased MiR-17 in glioma cells increased cell viability and migration by increasing the expression of Cyclin D1, p-Akt and Akt. PLoS One 13: e0190515, 2018.

20. Utaijaratrasmi P, Vaeteewoottacharn K, Tsunematsu T, Jamjantra P, Wongkham S, Pairojkul C, Khuntikeo N, Ishimaru N, Sirivatanauksorn Y, Pongpaibul A, et al: The microRNA-15a-PAI-2 axis in cholangiocarcinoma-associated fibroblasts promotes migration of cancer cells. Mol Cancer 17: 10, 2018.

21. Tahiri A, Aure MR and Kristensen VN: MicroRNA networks in breast cancer cells. Methods Mol Biol 1711: 55-81, 2018.

22. Lu G, Fu D, Jia C, Chai L, Han Y, Liu J, Wu T, Xie R, Chang Z, Yang H, et al: Reduced miR-105-1 levels are associated with poor survival of patients with non-small cell lung cancer. Oncol Lett 14: 7842-7848, 2017.

23. Mei LL, Qiu YT, Wang WJ, Bai J and Shi ZZ: Overexpression of microRNA-1470 promotes proliferation and migration, and inhibits senescence of esophageal squamous carcinoma cells. Oncol. Lett 14: 7753-7758, 2017.

24. Yang H, Wang L, Tang X and Bai W: miR-203a suppresses cell proliferation by targeting E2F transcription factor 3 in human gastric cancer. Oncol Lett 14: 7687-7690, 2017.

25. Kan Q, Su Y and Yang H: MicroRNA-335 is downregulated in papillary thyroid cancer and suppresses cancer cell growth, migration and invasion by directly targeting ZEB2. Oncol Lett 14: 7622-7628, 2017.

26. Hu B, Wang J and Jin X: MicroRNA-138 suppresses cell proliferation and invasion of renal cell carcinoma by directly targeting SOX9. Oncol Lett 14: 7583-7588, 2017.

27. Zhang G, Chen L, Khan AA, Li B, Gu B, Lin F, Su X and Yan J: miRNA-124-3p/neuropilin-1 (NRP-1) axis plays an important role in mediating glioblastoma growth and angiogenesis. Int $\mathrm{J}$ Cancer 143: 635-644, 2018.

28. Chen W, Kong KK, Xu XK, Chen C, Li H, Wang FY, Peng XF, Zhang Z, Li P, Li JL and Li FC: Downregulation of miR-205 is associated with glioblastoma cell migration, invasion, and the epithelial-mesenchymal transition, by targeting ZEB1 via the Akt/mTOR signaling pathway. Int J Oncol 52: 485-495, 2018.

29. Kong Y, Zou S, Yang F, Xu X, Bu W, Jia J and Liu Z: RUNX3-mediated up-regulation of miR-29b suppresses the proliferation and migration of gastric cancer cells by targeting KDM2A. Cancer Lett 381: 138-148, 2016.

30. Chen JY, Luo CW, Lai YS, Wu CC and Hung WC: Lysine demethylase KDM2A inhibits TET2 to promote DNA methylation and silencing of tumor suppressor genes in breast cancer. Oncogenesis 6: e369, 2017.

31. Huang Y, Liu Y, Yu L, Chen J, Hou J, Cui L, Ma D and Lu W: Histone demethylase KDM2A promotes tumor cell growth and migration in gastric cancer. Tumour Biol 36: 271-278, 2015.

32. Wagner KW, Alam H, Dhar SS, Giri U, Li N, Wei Y, Giri D, Cascone T, Kim JH, Ye Y, et al: KDM2A promotes lung tumorigenesis by epigenetically enhancing ERK1/2 signaling. J Clin Invest 123: 5231-5246, 2013.

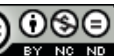

This work is licensed under a Creative Commons Attribution-NonCommercial-NoDerivatives 4.0 International (CC BY-NC-ND 4.0) License. 